\title{
Comparisons in Performance of Electromagnet and Permanent-Magnet Cylindrical Hall-Effect Thrusters
}

\author{
Kurt A. Polzin* \\ NASA-Marshall Space Flight Center, Huntsville, AL 35812 \\ Yevgeny Raitses, Jean Carlos Gayoso and Nathaniel J. Fisch ${ }^{\ddagger}$ \\ Princeton Plasma Physics Laboratory, Princeton, NJ 08543
}

\begin{abstract}
Three different low-power cylindrical Hall thrusters, which more readily lend themselves to miniaturization and low-power operation than a conventional (annular) Hall thruster, are compared to evaluate the propulsive performance of each. One thruster uses electromagnet coils to produce the magnetic field within the discharge channel while the others use permanent magnets, promising power reduction relative to the electromagnet thruster. A magnetic screen is added to the permanent magnet thruster to improve performance by keeping the magnetic field from expanding into space beyond the exit of the thruster. The combined dataset spans a power range from 50-350 W. The thrust levels over this range were 1.3-7.3 mN, with thruster efficiencies and specific impulses spanning 3.5-28.7\% and 400-1940 s, respectively. The efficiency is generally higher for the permanent magnet thruster with the magnetic screen, while That thruster's specific impulse as a function of discharge voltage is comparable to the electromagnet thruster.
\end{abstract}

\section{Introduction}

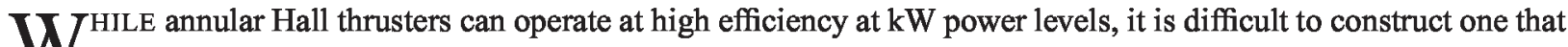
operates over a broad envelope from $\sim 1 \mathrm{~kW}$ down to $\sim 100 \mathrm{~W}$ while maintaining an efficiency of $45-55 \%$. Scaling to low power while holding the main dimensionless parameters constant requires a decrease in the thruster channel size and an increase in the magnetic field strength. ${ }^{1,2}$ Increasing the magnetic field becomes technically challenging since the field can saturate the miniaturized inner components of the magnetic circuit and scaling down the magnetic circuit leaves very little room for magnetic pole pieces and heat shields. In addition, the central magnetic pole piece defining the interior wall of the annular channel can experience excessive heat loads in a miniaturized Hall thruster, with the temperature eventually exceeding the Curie temperature of the material ${ }^{2}$ and in extreme circumstances leading to accelerated erosion of the channel wall.

An alternative approach is to employ a cylindrical Hall thruster (CHT) geometry. ${ }^{3}$ Laboratory model CHTs have operated at power levels ranging from $\sim 50 \mathrm{~W}$ up to $\sim 1 \mathrm{~kW}$. These thrusters exhibit performance characteristics that are comparable to conventional, annular Hall thrusters of similar size. Compared to the annular Hall thruster, the CHT's insulator surface area to discharge chamber volume ratio is lower. Consequently, there is the potential for reduced wall losses in the channel of a CHT, and any reduction in wall losses should translate into lower channel heating rates and reduced erosion, making the CHT geometry promising for low-power applications. This potential for high performance in the low-power regime has served as the impetus for research and development efforts aimed at understanding and improving CHT performance. ${ }^{3-7}$

In this paper, we present performance measurements from three separate variations on the CHT. Each variation is different in the manner in which the magnetic field is applied to the discharge channel. The use of electromagnets in one thruster offers the capability to test over a wide range of magnetic field strengths. The thrusters fabricated with permanent magnets do not offer this flexibility, but they do have the promise of reduced overall power consumption relative to the electromagnet variant. The use of permanent magnets simplifies the design by removing the multi-turn

\footnotetext{
*Propulsion Research Engineer, Propulsion Research and Technology Applications Branch, Propulsion Systems Department. Senior Member AIAA.

$\dagger_{\text {Research Physicist. Associate Fellow AIAA. }}$

$\ddagger$ Professor, Astrophysical Sciences Dept. Senior Member AIAA.
} 
electromagnet coils from the thruster. Thruster performance measurements (thrust, $I_{\mathrm{sp}}$, and anode efficiency) and the discharge current as a function of voltage are presented to compare the performance of the different thrusters.

\section{Experimental Apparatus}

All cylindrical Hall thruster testing presented in this paper was conducted at NASA's Marshall Space Flight Center (MSFC). We proceed first with a description of the three different thrusters and then discuss the facilities at MSFC.

\section{A. CHT with Electromagnet Coils}

Performance measurements obtained for the 3-cm electromagnet CHT (CHTem) shown in Fig. 1 were previously reported in Ref. [6]. The thruster consists of a boron-nitride ceramic channel, an annular anode, two electromagnet coils, and a magnetic core. The thruster channel is a composite of a shorter, annular region and a longer, cylindrical region. Gas is injected through the anode into the short, annular region of the thruster. The length of the annular region is sized to be greater than the ionization mean free path for xenon. This allows for high ionization of the propellant at the boundary between the annular and cylindrical regions. The electromagnet coils are operated using independently controlled power supplies, and the resulting field topology has a mirror-like structure near the thruster axis.

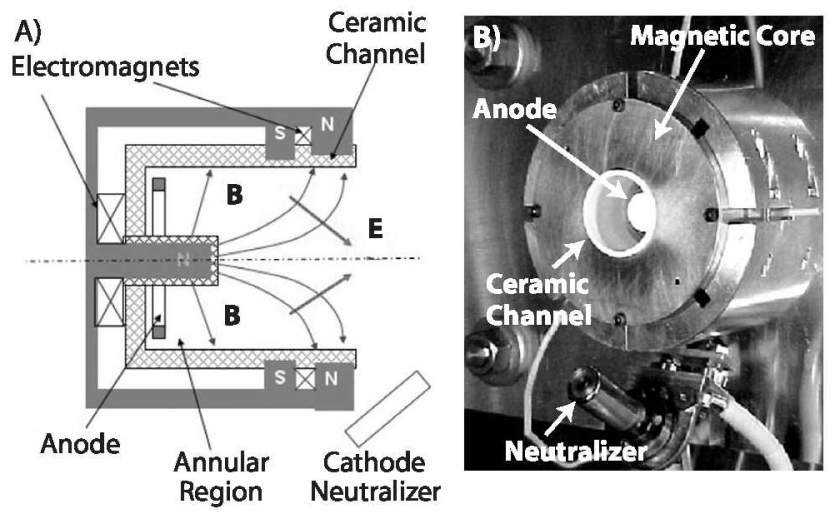

Figure 1. A) Schematic illustration and B) photograph of the 3-cm electromagnet cylindrical Hall thruster.

\section{B. CHT with Permanent Magnets}

Various measurements on the 2.6-cm channel diameter permanent magnet CHT (CHTpm-1) shown in Fig. 2a were previously reported in Refs. [8,9]. The thruster is roughly $5.5 \mathrm{~cm}$ in overall diameter and $3.5 \mathrm{~cm}$ long, massing roughly $350 \mathrm{~g}$. The thruster channel is comprised of a ceramic boron-nitride insulator with propellant fed from an annular anode. Unlike the CHTem, this thruster is purely cylindrical, possessing no shorter, annular region. Two sets of samarium-cobalt ( $\mathrm{Sm}-\mathrm{Co}$ ) rare-Earth magnets are used to produce the magnetic field. The magnets are oriented in the same direction to produce the 'direct' magnetic field topology shown in Fig. $2 \mathrm{~b}$ and the maximum field strength inside the thruster channel is roughly $1 \mathrm{kG}$. The cathode is oriented such that the orifice is $1.9 \mathrm{~cm}(0.75$ ") downstream of the thruster exit and $5.1 \mathrm{~cm}$ (2") from the thruster centerline. The cathode is aligned such that its centerline forms a $40^{\circ}$ angle with the thruster centerline.

\section{CHT with Permanent Magnets and a Magnetic Screen}

Recent measurements were obtained on the $2.6-\mathrm{cm}$ channel diameter permanent magnet CHT (CHTpm-2) shown in Fig. 3. This is the same thruster that described in the previous section, except it additionally possesses an external magnetic screen to keep the magnetic field from expanding into space downstream of the thruster. The thruster is operated with the cathode in one of two positions, with the orifice radially offset $1.47 \mathrm{~cm}$ in the minimum radial position and $2.35 \mathrm{~cm}$ in the 'zero' position. In both cases, the cathode is oriented perpendicular to the thruster axis and the orifice is located roughly $2 \mathrm{~cm}$ downstream of the thruster exit plane. 
a)

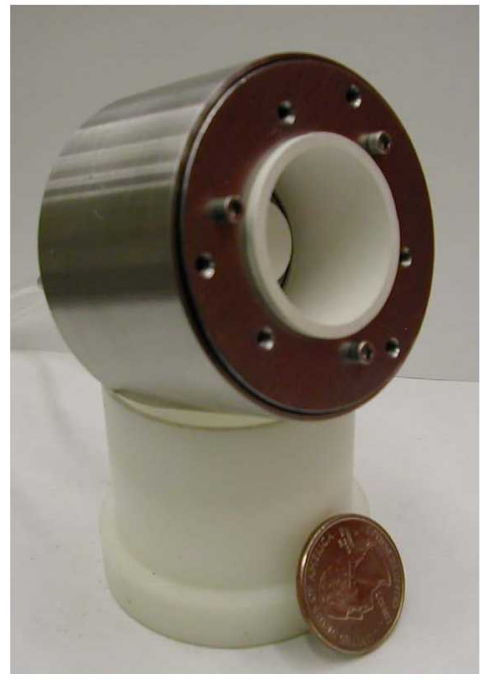

b)

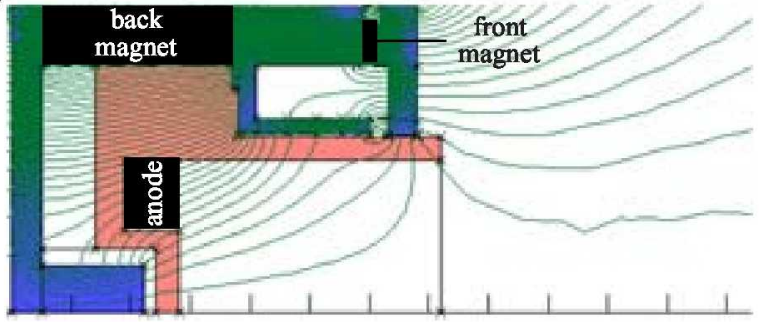

Figure 2. a) Laboratory model 2.6-cm CHT with Sm-Co permanent magnets (with US quarter for scale). b) Magnetic field topology in a laboratory model $2.6 \mathrm{~cm}$ CHT with Sm-Co permanent magnets where the magnets are oriented in a direct-field alignment. The maximum magnetic field is roughly $1 \mathrm{kG}$ at the axis near the back wall.

a)

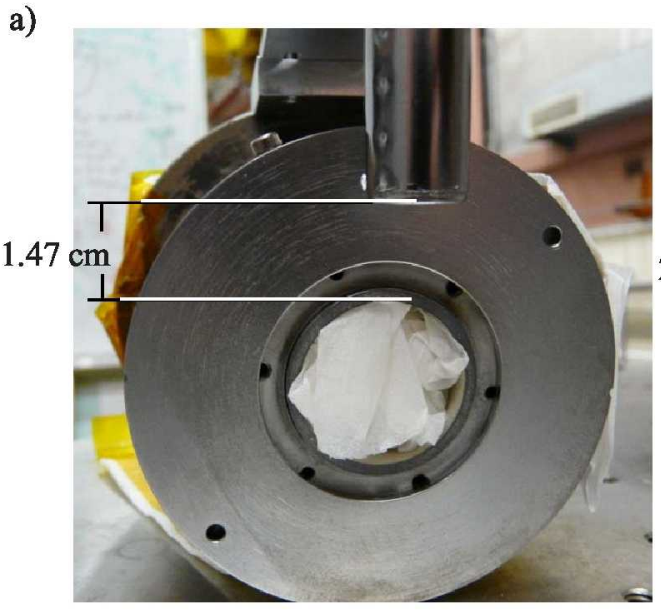

b)

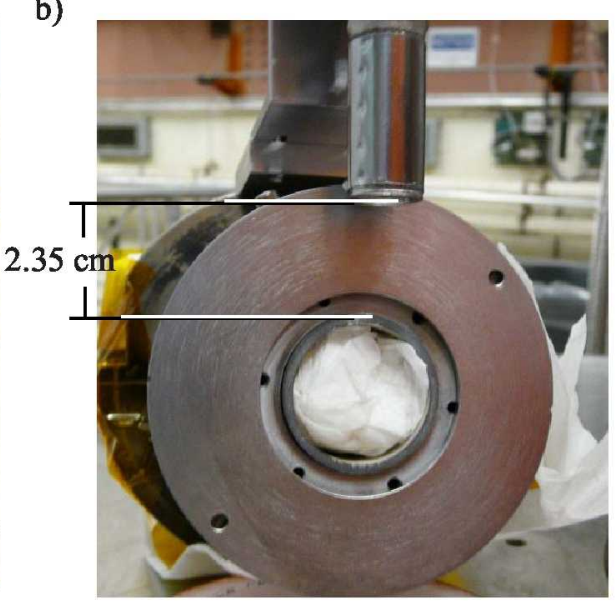

Figure 3. Laboratory model 2.6-cm CHT with Sm-Co permanent magnets and a magnetic screen outer shell with the cathode a) in the minimum radial position and b) in the 'zero' position. c) Magnetic field map of the thruster.

\section{NASA-MSFC Test Facility}

In all three cases a commercial HeatWave Labs HWPES-250 hollow cathode is used, serving as both the thruster cathode and the beam neutralizer. The working propellant for all experiments is research-grade xenon gas, and the cathode and anode flow rates are independently controlled. The propellant flow rate to both the cathode and anode were controlled using two variable 10-sccm MKS 1479 precision flow controllers (calibrated on Xe and controllable to $\pm 0.1 \mathrm{sccm}$ ). All testing was performed with a cathode flow rate of $2 \mathrm{sccm}$. Power was provided to the thruster and cathode using Sorenson switching power supplies.

Testing was conducted in a $2.75-\mathrm{m}$ diameter, $7.6-\mathrm{m}$ long stainless steel vacuum chamber. The vacuum level inside the chamber is maintained by two $9500 \mathrm{l} / \mathrm{s}$ gaseous helium cryopumps. The base pressure of the facility was $2 \times 10^{-7}$ torr, and the pressure level (corrected for Xe) during testing was roughly $1-2 \times 10^{-5}$ torr.

Thrust was measured using the variable-amplitude hanging pendulum with extended range (VAHPER) thrust stand. ${ }^{10}$ The stand employs a unique linkage mechanism to convert horizontal deflection of the pendulum arm into amplified vertical deflection of a secondary beam. Displacement (thrust) calibration of the VAHPER thrust stand is accomplished using an in situ calibration rig that applies a series of known loads normal to the pendulum arm. Cal- 
ibration can be performed before, during, and after thruster operation. The measured displacement of the vertically deflecting linkage is recorded as the calibration loads are applied to the arm. Assuming that the relationship between the applied force and the measured displacement is linear allows for a linear curve fit of the calibration data.

It should be noted that there are still some issues that remain to be explored, especially with CHTpm-2. The most important is the fact that, for a given discharge current, the thruster draws more current in the MSFC setup than in the test setup at the Princeton Plasma Physics Laboratory (PPPL). It is unclear at this time how important this effect is with respect to the integrity of the measured data. It also needs to be explored in terms of whether the source of this discrepancy resides in the test setup (cathode mount position, etc.) or is a facility effect.

\section{Experimental Data}

Thrust, thruster efficiency, and specific impulse $\left(I_{\mathrm{sp}}\right)$ measurements for all the thrusters described in the previous section are presented in Fig. 4. The thrust and specific impulse measurements are given according to their standard definitions. ${ }^{11}$ To account for the added power required by the CHTem relative to the CHTpm models, the thruster efficiency is calculated as the ratio of the exhaust jet power to the sum of the discharge power and the electromagnet power. For the CHTem, the power applied to the electromagnet coils is roughly $100 \mathrm{~W}$. CHTpm-1 and -2 are not penalized by this additional power loss because they do not have electromagnet coils. The data for CHTem and CHTpm-1 are displayed in Figs. 4a-c, while the data for CHTpm-2 operating with the cathode exit located at the minimum radius and 'zero' positions are shown in Figs. 4d-f.
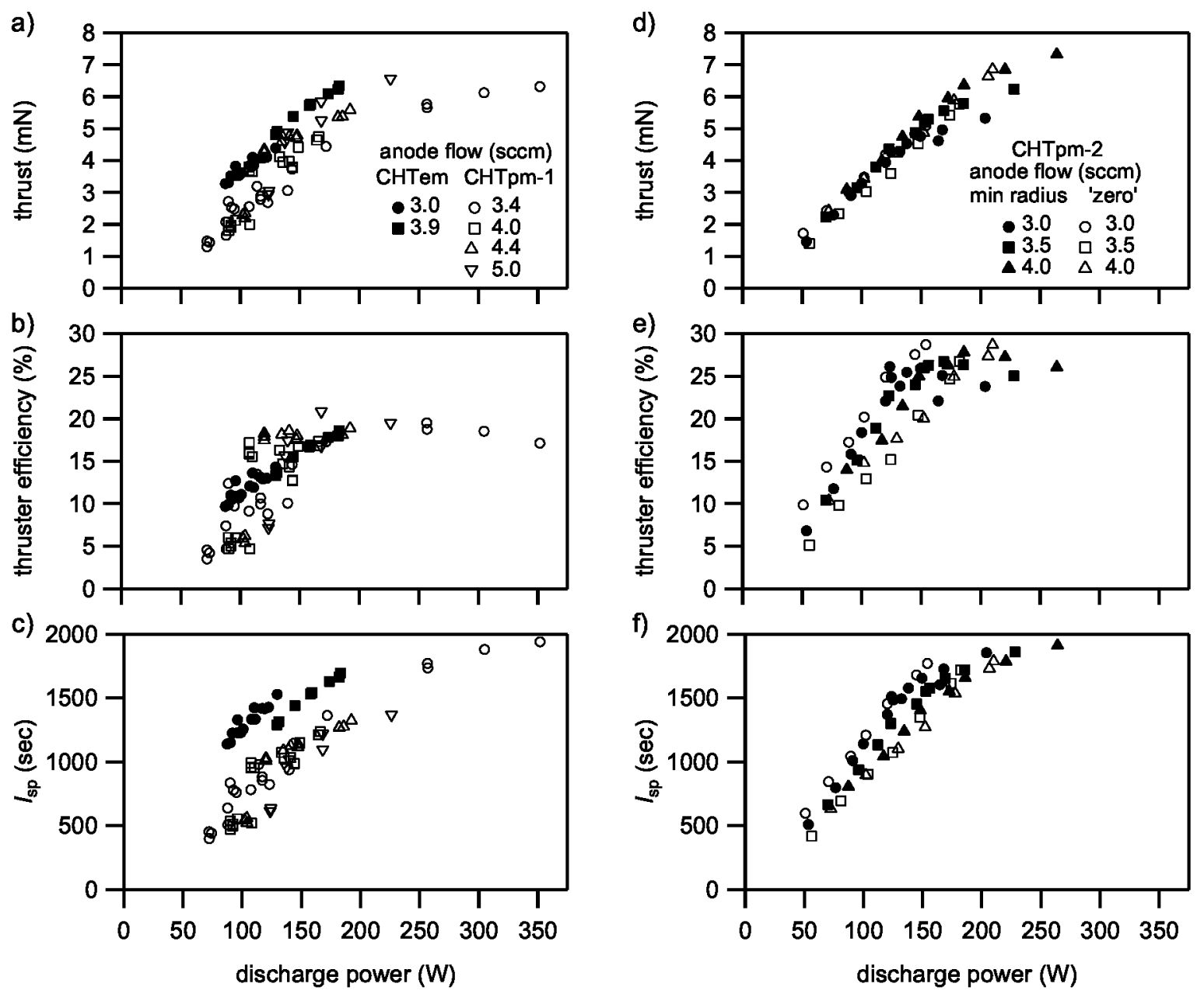

Figure 4. Thrust, thruster efficiency (including electromagnet power consumption for CHTem), and $I_{\text {sp }}$ as a function of discharge power for a)-c) the CHTem and CHTpm-1 and d)-f) the CHTpm-2 for both cathode positions (minimum radius and 'zero'). The average error bars (with a $95 \%$ confidence interval) on these data are roughly $150 \mu \mathrm{N}, 1.1 \%$, and $40-50$ sec. 
The data span the range from 50-350W in discharge power, resulting in thrust levels between 1.3 and $7.3 \mathrm{mN}$,

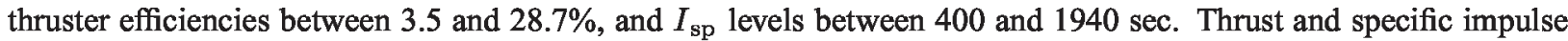
increase with discharge power in all cases. Thruster efficiency increases with discharge power below $200 \mathrm{~W}$, but in the instances where data are available the efficiency starts to asymptote or even decrease above $200 \mathrm{~W}$.

The performance data show that the CHTpm-2 with the magnetic screen installed generally has greater efficiency for both cathode positions. Below $150 \mathrm{~W}$ with the cathode at the minimum radius position the efficiency at a given discharge power level is roughly the same for all three flow rates tested. Just as interesting is that the efficiency at the low power level of $50 \mathrm{~W}$ and a flow rate of $3.0 \mathrm{sccm}$ is $9.9 \%$ While the CHTem has lower efficiency relative to CHTpm-2, it should be noted that the thrust and $I_{\mathrm{sp}}$ of the former are higher and it is only the electromagnet power that reduces the efficiency of the CHTem to the level reported here. At low discharge power levels, there is a higher degree of variability and spread in the specific impulse and efficiency of CHTpm-1 relative to the other data sets. During testing the operation of CHTpm-1 at lower discharge power levels was much more sensitive to very small changes in the discharge parameters relative to the other thrusters, producing the inconsistent or scattered results observed in the data set.
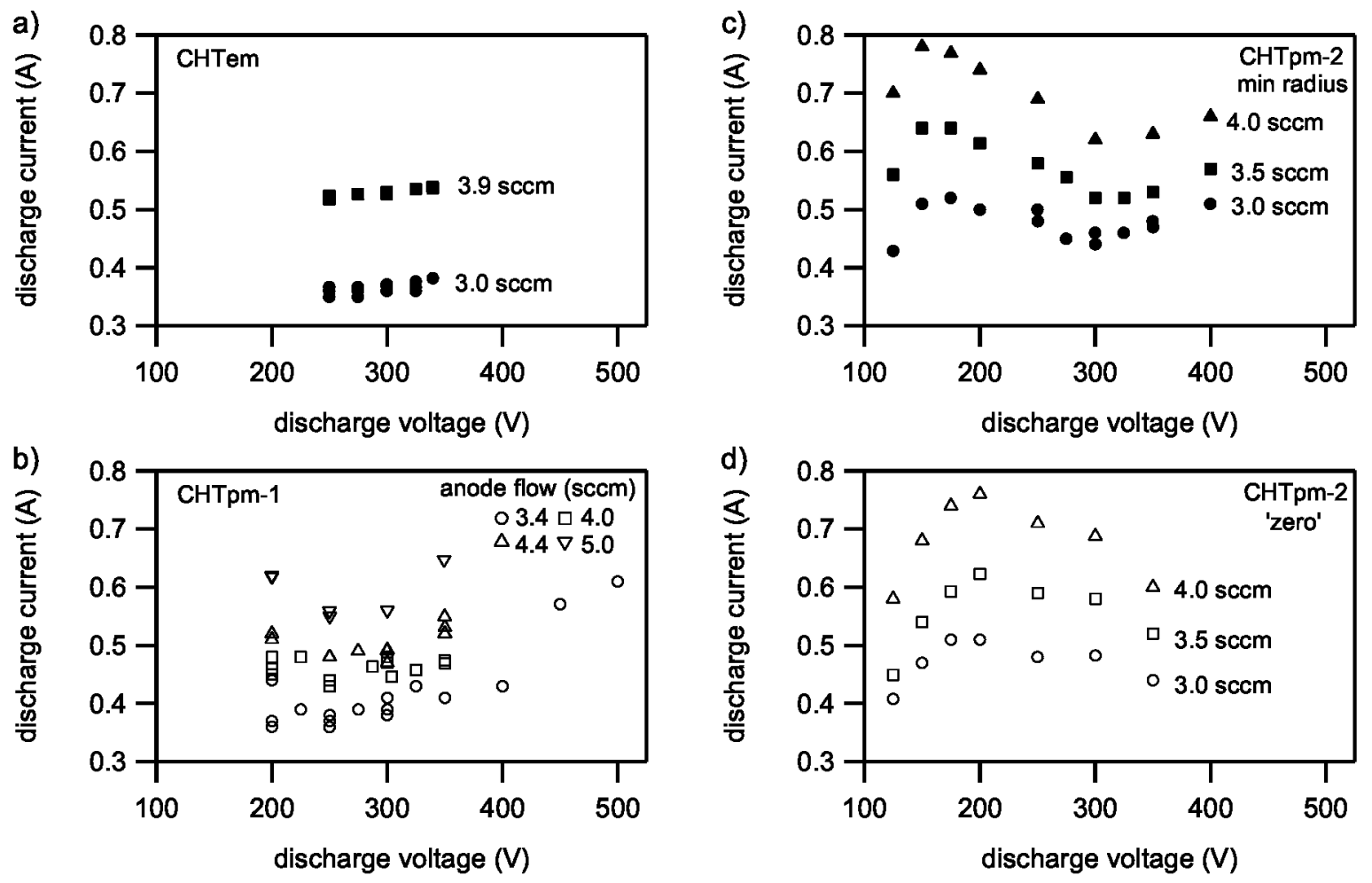

Figure 5. Discharge current as a function of discharge voltage and anode flow rate for a) CHTem, b) CHTpm-1, c) CHTpm-2 (minimum radius cathode position), and d) CHTpm-2 ('zero' cathode position).

The discharge current as a function of voltage for all thruster configurations is presented in Fig. 5. For a given thruster, we observe that in all cases the current is greater at higher flow rates. While only a small span of discharge voltages was tested using the CHTem, the data over this span exhibit a relatively constant discharge current. Except for the lowest flow rate, the discharge current for CHTpm-1 exhibits a local minimum between 250 and $300 \mathrm{~V}$. While the current levels for CHTem and CHTpm-1 are somewhat comparable, the currents drawn by CHTpm-2 represent a significant increase. Functionally, both CHTpm-2 data sets follow the same basic form, exhibiting a local maxima at lower voltages. While the discharge in the CHTpm-2 extinguished above $350 \mathrm{~V}$ in the 'zero' configuration, the other cathode position did permit operation at higher voltages and resulted in the appearance of local minima in the discharge current.

The $I_{\mathrm{sp}}$ as a function of discharge voltage is given in Fig. 6 for all thruster configurations. In all cases, $I_{\mathrm{sp}}$ increases with discharge voltage. In most cases, $I_{\mathrm{sp}}$ is higher at higher flow rates. However, for CHTpm-2 with the cathode in the 'zero' position, the lowest specific impulse at a given discharge voltage occurs at $3.5 \mathrm{sccm}$, with higher $I_{\mathrm{sp}}$ at both 3.0 and $4.0 \mathrm{sccm}$. From 250-350 V, the specific impulse of CHTem and CHTpm-2 are comparable in magnitude. 

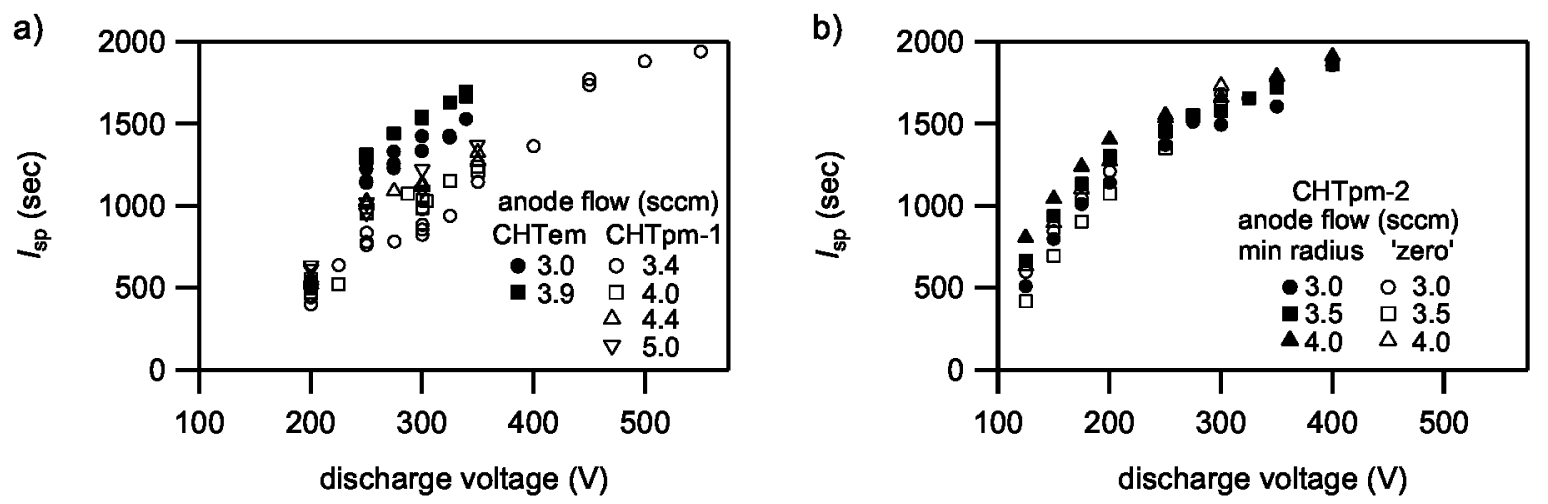

Figure 6. $I_{\mathrm{sp}}$ as a function of discharge voltage for a) the CHTem and CHTpm-1, b) the CHTpm-2 for both cathode positions (minimum radius and 'zero').

The effect of the magnetic screen on the permanent magnet thruster performance is clearly seen in the upwards shift in specific impulse for CHTpm-2 relative to CHTpm-1 at a given discharge voltage.

For those cases where at a given voltage $I_{\mathrm{sp}}$ is greater for a given mass flow rate, we can speculate that this increase is due to greater propellant use (i.e., low neutral fraction), the formation of doubly ionized Xe, or a combination of the two. However, we do not have enough information at this time to reach a definite conclusion.

\section{Conclusions}

Although conventional (annular) Hall thrusters are efficient in the kilowatt power regime, they become inefficient when scaled to small sizes. This is due to the difficulties associated with holding the performance scaling parameters constant while decreasing the channel size and increasing the applied magnetic field strength. The cylindrical Hall thruster can be more readily scaled to smaller sizes due to its nonconventional discharge-chamber geometry and associated magnetic field profile.

In the present paper, a series of measurements obtained on three separate cylindrical Hall thrusters were compared. One thruster employed an electromagnet coil to generate the magnetic field in the thruster while the other two used permanent magnets. The use of permanent magnets promises lower thruster power consumption by eliminating the electromagnet power sink. In addition, permanent magnets simplify the design, removing the need for additional power supplies and multi-turn electromagnet coils.

Performance measurements for all thrusters were obtained using the VAHPER thrust stand. The combined data span the range of discharge power from 50-350 W. The thruster produced thrust levels ranging from 1.3-7.3 $\mathrm{mN}$, thruster efficiencies spanning 3.5-28.7\%, and $I_{\mathrm{sp}}$ between 400-1940 sec. All performance parameters generally increased with discharge power, except in testing of the CHTpm-1 above $250 \mathrm{~W}$. For that thruster above $250 \mathrm{~W}$, the thruster efficiency was reduced with increasing discharge power. The performance data show a generally higher efficiency for CHTpm-2 for both cathode positions, with the capability to operate at lower power levels. The $I_{\mathrm{sp}}$ as a function of discharge voltage was comparable between CHTem and CHTpm-2, while the specific impulse on CHTpm1 was lower, indicating the positive effect of the magnetic screen on the performance of the permanent magnet thruster.

\section{Acknowledgments}

Work performed by the Princeton Plasma Physics Laboratory coauthors was partially supported by the U.S. Air Force Office of Scientific Research. We gratefully acknowledge the contributions of Enrique Merino, who contributed technical assistance in the design of the magnetic screen, and to MSFC technical support staff Tommy Reid and Doug Galloway. We extend our thanks to students Adam Kimberlin, Ryan Sullenberger, Valerie Hanson, Lindsey Walker, and Kevin Bonds for their efforts. We also appreciate and acknowledge the continued MSFC support of Mr. James Martin, Mr. J. Boise Pearson, Dr. Thomas Brown, and Mr. Thomas Williams. 


\section{References}

${ }^{1}$ A.I. Morozov and V.V. Savelyev, "Fundamentals of Stationary Plasma Thruster Theory," Reviews of Plasma Physics, edited by F.F. Kadomtsev and V.D. Shafranov, Vol. 21, Consultants Bureau, New York, 2000, p. 203.

${ }^{2}$ V. Khayms and M. Martinez-Sanchez, "Fifty-Watt Hall Thruster for Microsatellites," Micropropulsion for Small Spacecraft, edited by M.M. Micci and A.D. Ketsdever, Vol. 187, Progress in Astronautics and Aeronautics, AIAA, Reston, VA, 2000, pp. 233-254.

${ }^{3}$ Y. Raitses and N.J. Fisch, "Parametric Investigations of a Nonconventional Hall Thruster," Phys. Plasmas, 8(5):2579, 2001.

${ }^{4}$ A. Smirnov, Y. Raitses, and N.J. Fisch, "Parametric Investigations of Miniaturized Cylindrical and Annular Hall Thrusters," J. Appl. Phys., 92(10):5673, 2002.

${ }^{5}$ A. Smirnov, Y. Raitses, and N.J. Fisch, "Plasma Measurements in a 100 W Cylindrical Hall Thruster," J. Appl. Phys., 95(5):2283, 2004.

${ }^{6}$ K.A. Polzin, T.E. Markusic, B.J. Stanojev, A. Dehoyos, Y. Raitses, A. Smimov, and N.J. Fisch, "Performance of a Low-Power Cylindrical Hall Thruster," J. Propuls. Power, 23(4):886, 2007.

${ }^{7}$ Y. Raitses, E. Granstedt, A. Smirnov, E. Merino, and N.J. Fisch, "Effects of Cathode Electron Emission on Hall Thruster Discharge," AIAA Paper 2008-5188, July 2008.

${ }^{8}$ K.A. Polzin, E.S. Sooby, A.C. Kimberlin, Y. Raitses, E. Merino, and N.J. Fisch, "Performance of a Permanant-Magnet Cylindrical Hall-Effect Thruster," AIAA Paper 2009-4812, Aug. 2009.

${ }^{9}$ K.A. Polzin, E.S. Sooby, Y. Raitses, E. Merino, and N.J. Fisch, "Discharge Oscillations in a Permanent Magnet Cylindrical Hall-Effect Thruster," 31st International Electric Propulsion Conference, Ann Arbor, MI, IEPC Paper 2009-122, Aug. 2009.

${ }^{10}$ K.A. Polzin, T.E. Markusic, B.J. Stanojev, A. DeHoyos and B. Spaun, "Thrust Stand for Electric Propulsion Performance Evaluation," Rev. Sci. Instrum., 77:105108, 2006.

${ }^{11}$ R.G. Jahn, Physics of Electric Propulsion, McGraw-Hill, New York, 1968, pp. 4, 7. 